\title{
Variation of Generation Times in Escherichia coli Populations: its Cause and Implications
}

\author{
By HANS BREMER \\ The University of Texas at Dallas, P.O. Box 688, Richardson, Texas 75080, U.S.A.
}

(Received 22 March 1982; revised 20 May 1982)

\begin{abstract}
The cell cycle of Escherichia coli contains a period of indeterminate length that reflects a stochastic reaction, beginning at some time after a round of chromosome replication, and ending before the cell divides. Although the chemical nature of this reaction is not known, the time of its onset and the single statistical 'half-life parameter' required for its quantitative description have been measured previously. Here it is shown that this parameter implies the distribution of generation times and the age distribution, as well as the distributions of replication initiation and termination ages; these distributions are derived from this half-life parameter for exponentially growing populations of $E$. coli. It is also shown that the stochastic reaction affects the results and interpretation of any experiments involving synchronous growth of bacteria.
\end{abstract}

\section{INTRODUCTION}

The distribution of generation times has been studied in various genera of bacteria by microscopic observation of individual cells growing either in a liquid culture on a membrane through which growth medium was circulated (Powell, 1956, 1958; Powell \& Errington, 1963; Schaechter et al., 1962), or on solid medium (Kubitschek, 1961). By fitting the observed generation time distributions, which showed considerable scatter, to various theoretical distributions, the values of statistical parameters were obtained, such as standard deviation, coefficient of variation, skewness and kurtosis. The biological meaning of these parameters, however, was not clear. With regard to the skew in the distributions, the results were ambiguous; some data fitted best to a symmetrical distribution (Schaechter et al., 1962), while others suggested a skew toward longer generation times (most of E. O. Powell's work; Kubitschek, 1961). Generation time distributions have also been derived (Shehata \& Marr, 1970; Harvey, 1972) from growth curves of synchronous bacterial cultures (Helmstetter \& Cooper, 1968), thus avoiding many statistical problems of the methods based on observation of individual cells. From such data, symmetrical distributions of generation times were obtained.

The most striking feature of the distribution of generation times is the extent of variation; for example, at an average doubling time of $40 \mathrm{~min}$, early divisions may occur $20 \mathrm{~min}$ after, and late divisions more than $60 \mathrm{~min}$ after the preceding division. Such a range of generation times in different cells of the same population must be taken into account in any model of the control of bacterial division.

We have recently analysed the cell cycle of Escherichia coli (Bremer \& Chuang, 1981 a,b) and reduced the cell cycle variability to a variability of the $D$-period, the time between termination of a round of replication and the following cell division. The type of variability observed suggested the involvement of a stochastic reaction in the division pathway, presumably in the initiation of septum formation. In the present paper, it is shown that the statistical properties of bacterial populations that have been measured in the past depend on, and can be quantitatively derived from, this variability in the $D$-period. The question about the skew in the generation time distributions is also resolved: there is not a single distribution of generation times valid for all 
populations; rather, depending on the type of population considered, skewed or symmetrical distributions are to be expected. It is further shown that the variability in the $D$-period has important implications for any work with synchronous bacterial cultures; therefore, the theory derived here should be taken into account in the design and interpretation of synchronous growth experiments.

\section{THEORY AND DISCUSSION}

\section{Variability of the D-period}

By measuring cell numbers and the rate of DNA synthesis in synchronous cultures of $E$. coli $\mathrm{B} / \mathrm{r}$, we have previously shown that the duration of the $D$-period varies in a population of cells; this variation is described by a frequency distribution as in Fig. $1:$ the minimum $D$-period, $D_{0}$, is $18 \mathrm{~min}$, while $D$-periods longer than $18 \mathrm{~min}$ occur with a frequency that decreases exponentially with increasing duration (Bremer \& Chuang, 1981a). In these experiments, the half-life time for the exponential decrease, $h$, was about $5 \mathrm{~min}$. The asymmetric distribution of $D$-periods was confirmed by analysing residual cell divisions after inhibition of DNA replication in exponential cultures (Bremer \& Chuang, 1981b). In this instance, the half-life value was about $10 \mathrm{~min}$; the difference in $h$-values was ascribed to the thy $A$ mutation in the strain used for the later experiments. The distribution in Fig. 1 is for a thymine-requiring strain of $E$. coli $\mathrm{B} / \mathrm{r}$ grown at $37^{\circ} \mathrm{C}$ in glucose minimal medium with an excess of thymine (Bremer \& Chuang, $1981 b)$. The average $D$-period, $\bar{D}$, is the sum $D_{0}+(h / \ln 2)$, where $h / \ln 2$ is the time for the exponential curve to decrease to $1 /$ e. In Fig. $1, \bar{D}=18+(10 / \ln 2)=32 \cdot 4 \mathrm{~min}$. This average is somewhat longer than the value of $D$ used for most calculations regarding the cell cycle (see below: Average values of cell cycle parameters).

The sharp peak in the distribution of $D$-periods is an idealization, which allows the distribution to be described by an exponential function that can be mathematically manipulated. The actual blurring of this peak extends over a period of a few minutes (Bremer \& Chuang, 1981 $a$; see also Fig. 3 of Gudas \& Pardee, 1974). This idealization does not significantly affect observable properties of bacterial cultures, which depend on the integral of the distribution in Fig. 1.

The variability of the $D$-periods could mean either (a) that the time between termination of rounds of replication and initiation of septation is constant, but the time required for completion of septation ( $T$-period) varies or (b) that the $T$-period is constant but the onset of septation varies. Experimental evidence suggests that the $T$-period is constant (e.g. Gudas \& Pardee, 1974; Woldringh et al., 1977), suggesting that the stochastic process affects the initiation of septum formation. The idea that there exists a period of indeterminate length before DNA replication in the cell cycle (Smith \& Martin, 1973) is thus at variance with the data for $E$. coli in which the stochastic reaction occurs after DNA replication (Bremer \& Chuang, 1981 $a, b$ ).

For the following theoretical derivations, it is assumed that no other factors contribute to the variability of the cell cycle besides the variability of $D$-periods. In particular, it is assumed that

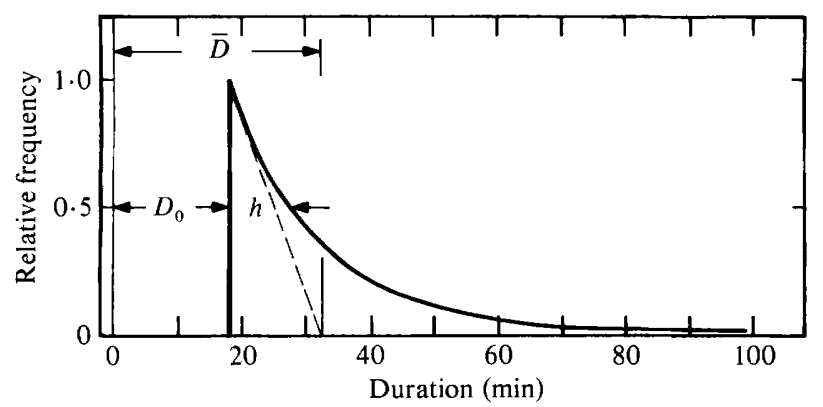

Fig. 1. Idealized frequency distribution of $D$-periods in $E$. coli $\mathbf{B} / \mathrm{r}$ (from Bremer \& Chuang, $1981 b$ ). Zero time is the time of termination of a round of replication. $D_{0}=\operatorname{minimum} D$-period, $\bar{D}=$ average $D$-period, $h=$ half-life time. 
neither the interinitiation times (time from initiation of a round of replication to initiation of the next round), nor the $C$-period (time from initiation to termination of a round of replication) vary significantly from cell to cell within a population or from cycle to cycle in successive generations. Experimental evidence justifying these assumptions has been discussed (Bremer \& Chuang, $1981 a$ ).

An invariability of those other cell cycle parameters implies that the fluctuations of growth and DNA replication in a synchronous culture (no matter how 'good' or 'bad' the synchrony) that occur during the first generation of synchronous growth should repeat themselves indefinitely in exactly the same manner without blurring. Kepes \& Kepes (1980) have followed synchronous cultures of $E$. coli for more than four generations and showed that the loss of synchrony is indeed very small during this time.

\section{Composition of a zero age population from an exponential culture}

As a consequence of the variability of $D$-periods, individual cells in a zero-age population of bacteria would be heterogeneous with respect to the time that the preceding round of replication was terminated. Cells having a long $D$-period $\left(>D_{0}\right)$ would grow more and be larger than those dividing at $D_{0}$. Thus, subfractionating a zero-age population of bacteria by size would yield different cell populations that are out of step with respect to their replication cycles, but in step with respect to division. In the past, synchronous cultures have been prepared either by size fractionation (Mitchison \& Vincent, 1965) or by age fractionation (Helmstetter \& Cummings, 1964). These types of synchronous cultures are expected to have different compositions with respect to the phases of their chromosome replication cycles and therefore to behave differently (see below). In the following, the distribution of the replication age (i.e. the cell age measured from the time of termination of replication) is derived for the entire, unfractionated zero-age population of an exponential culture. This is actually a hypothetical case since no reported method is capable of extracting all subpopulations of zero-age cells from an exponential culture with equal probability and without contamination by non-zero-age cells (Bremer \& Churchward, 1978).

Figure 2 shows the increase with time in the number of chromosomal replication termini $(T)$ and of the cell number $\left(C_{t}\right)$ in an exponential culture, here assumed to be growing with a doubling time $\tau=45 \mathrm{~min}$. At an arbitrarily chosen zero time, the cell number in this culture was set at unity. The infinitesimal increment in the cell number at zero time, $\mathrm{d} C_{0}$, from $t=0$ to $t=$ $\mathrm{d} t$, represents the increment due to new-born cells. This zero-age population is composed of subpopulations of cells that have terminated a round of replication at different times before zero

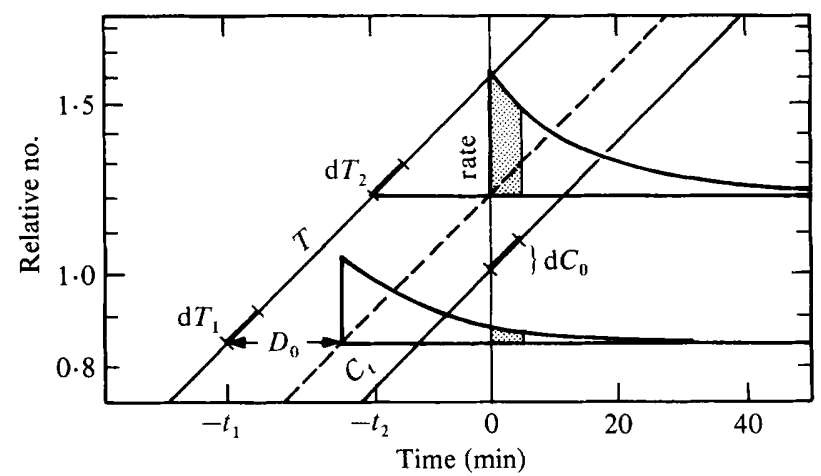

Fig. 2. Relationship between times of replication termination and the rate of cell division in an exponential culture. $\mathrm{d} T_{1}$ and $\mathrm{d} T_{2}$ represent the numbers of cells in which rounds of replication were terminated at times $-t_{1}$ and $-t_{2}$ before zero time; these cells define two subpopulations of the exponential culture that contribute to the rate of division at zero time (cell number increment $=\mathrm{d} C_{0}$ ). The stippled areas under the cell division rate curves of the two subpopulations represent the contributions of these subpopulations to the rate of cell division in the exponential culture at $t=0 . T=$ numbers of chromosome termini, $C_{t}=$ cell number as a function of time. Note that the ordinate scales for the division rate curves are linear. See text for details. 


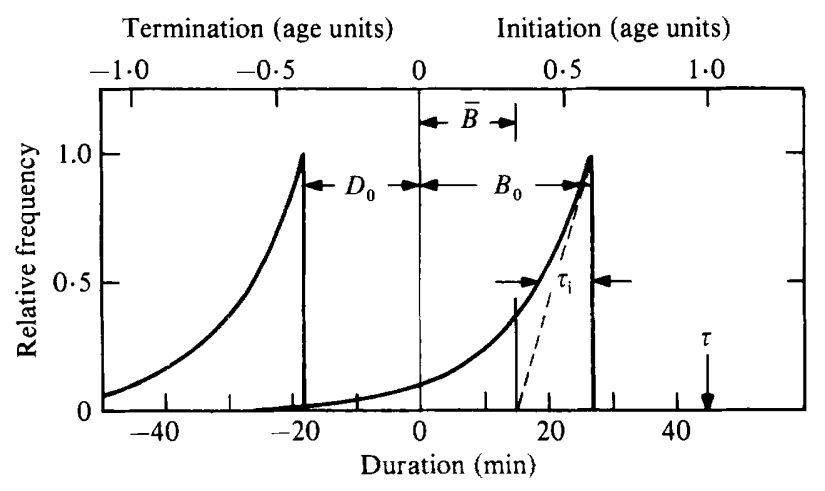

Fig. 3. Frequency distribution of termination times (left curve) and of initiation times (right curve) in a synchronous culture (all cells divide at zero time) derived from an exponential culture $(\tau=45 \mathrm{~min}, h=$ $10 \mathrm{~min}$; see text and equation (A2), Appendix). --- , Initial slope, extrapolates to $\bar{B}$ and the abscissa.

time, for example, at $t=-t_{1}$ and at $t=-t_{2}$ [i.e. between $-t_{1}$ and $\left(-t_{1}+\mathrm{d} t\right)$ and between $-t_{2}$ and $\left.\left(-t_{2}+\mathrm{d} t\right)\right]$; the numbers of cells in these subpopulations are $\mathrm{d} T_{1}$ and $\mathrm{d} T_{2}$. Each of these subpopulations begins to divide $D_{0} \mathrm{~min}$ later, and their contributions to the zero-time division rate are indicated by the stippled areas under the division rate curves for the corresponding subpopulations. The latest time at which terminations may occur that contribute to newborn cells at zero time is $-t_{2}\left(=-D_{0}\right)$. Cells in which replication terminates later (between $-t_{2}$ and zero) would not begin to divide until after zero time. Most of the new-born cells at zero time are derived from cells that have terminated a round of replication around or shortly before $-t_{2}$, while cells that have terminated earlier, e.g. at the time $-t_{1}$, contribute only few new-born cells at zero time. Thus, the frequency distribution of termination times before zero that contribute to the zero-age population increases exponentially until $-D_{0}(=18 \mathrm{~min})$ and then ends abruptly (Fig. 3, left curve).

The division rate curves associated with a given termination time $\left(-t_{1}\right.$ and $-t_{2}$ in Fig. 2) have the same shape as the distribution of $D$-periods in Fig. 1; the area under each rate curve, which represents the total number of cells in this subpopulation, is equal to $d T$. This area increases exponentially with time, corresponding to the exponential increase in the rate of termination in an exponential culture (doubling time $\tau$ ). Therefore, the fraction of cells that divide at zero time and that have terminated a given time $(-t)$ before zero time increases with increasingly later termination (up to $-D_{0}$ ) as the product of two exponential functions: $2^{t / \tau}$, which represents the increase due to the culture growth (barely visible in Fig. 2), and $2^{t / h}$, which results from the variability of the $D$-periods (major contribution to the diffference in the stippled areas under the two rate curves; $h$ is the half-life parameter defined in Fig. 1 ). This product, $2^{t / \tau}$ $\times 2^{t / h}$, is again an exponential function, $2^{t / \tau_{i}}\left(\right.$ where $\left.\left(1 / \tau_{\mathrm{j}}\right)=(1 / \tau)+(1 / h)\right)$, which describes the distribution of termination times in the zero-age population from an exponential culture (Fig. 3, left curve; Appendix A).

Cells that have terminated a round of replication early, before zero time, will initiate the following round of replication early also, and cells that terminated late will initiate late after zero time, since the times from initiation to the next initiation, and from termination to the next initiation, are essentially invariable (see above). Thus, the distribution of initiation times ( $B$ periods) can be represented by a curve (right curve in Fig. 3) that parallels the termination curve. Generally, the distributions of replication initiation and termination ages can be expected to be identical in shape but shifted along the time axis.

\section{Distribution of generation times in an exponential culture}

Based on previous theoretical work (Bremer \& Chuang 1981 a), the distribution of generation times was calculated from the distribution of termination times (Fig. 3) and from the 
distribution of $D$-periods (Fig. 1; see Appendix B for details). The result of this calculation, i.e. the distribution of generation times in an exponential culture, is shown in Fig. 4( $a, b)$, calculated for $\tau=43 \mathrm{~min}$ and $h=5 \mathrm{~min}$ (Fig. $4 a$ ), typical for $E$. coli $\mathrm{B} / \mathrm{r}$ growing in glucose minimal medium at $37^{\circ} \mathrm{C}$, and for $\tau=45 \mathrm{~min}$ and $h=10 \mathrm{~min}$ (Fig. $4 \mathrm{~b}$ ), typical for a thymine-requiring derivative of $E$. coli $\mathrm{B} / \mathrm{r}$ (Bremer \& Chuang, $1981 a, b)$. The distributions are seen to have a sharp peak at $t=\tau \mathrm{min}$ and to be slightly skewed toward longer generation times.

As indicated in the Introduction, the question of whether the distribution of generation times is skewed or symmetrical has been controversial. In our previous work, we found a symmetrical distribution of interdivision intervals (Bremer \& Chuang, 1981a). The apparent discrepancy reflects the different composition of the zero-age population considered. In the previous work, we derived the properties of a synchronous culture obtained from an inverted membrane culture in which all ages were equally frequent (see Fig. $6 c$ below), whereas here we considered a synchronous culture derived from the zero-age population of an exponential culture.

The extent of asymmetry or skew in the distribution of interdivision intervals can be expressed by the ratio of the time constants that describe the right $(h)$ and the left $\left(\tau_{\mathrm{i}}\right)$ limbs of the distribution, i.e. the ratio $h / \tau_{\mathrm{i}}$, equal to $1+h / \tau$ (see definition of $\tau_{\mathrm{i}}$ in the preceding section). The greater the value of $h / \tau$, the greater the asymmetry: $h / \tau=0$ means no skew. For $\tau=45 \mathrm{~min}$ and $h=10 \mathrm{~min}, h / \tau=0.22(10 / 45)$; with this value, the asymmetry is barely discernible [compare solid and dashed curves in Fig. 7(a) below], suggesting that most of the previously observed skew in the generation time distributions has resulted from environmental heterogeneity, e.g. within the microcolonies observed under the microscope.

\section{Age distribution of an exponential culture}

The age of a cell is defined as the time elapsed since the preceding division, measured in units of the culture doubling time; i.e. new-born cells have the age zero and, if the culture doubling time is $\mathbf{4 5} \mathrm{min}$, then cells that have not yet divided at $45 \mathrm{~min}$ after the preceding division would have the age $1 \cdot 0$. If all cells were to divide regularly in exactly the same intervals, e.g. every $45 \mathrm{~min}$, then the oldest cells in the population would have the age 1.0 , and thus the age distribution would abruptly end at 1.0. The age distribution from such a hypothetical

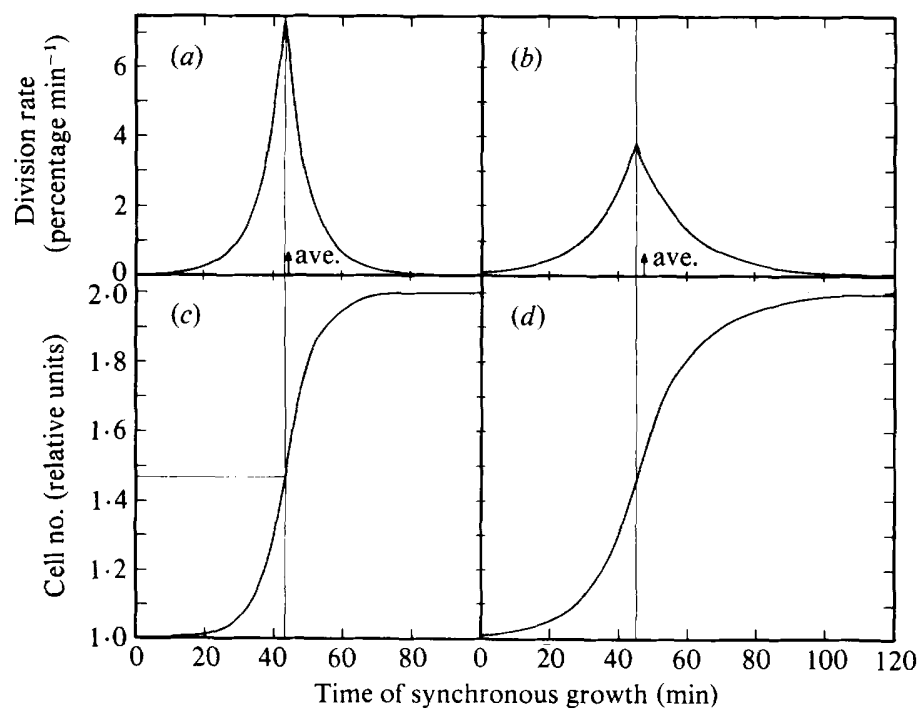

Fig. 4. Rate of division (top panels) and cell number (bottom panels) during one cycle of synchronous growth of a zero-age population from an exponential culture. [Division rates were calculated using equations (B2) and (B3); cell numbers were calculated using equations (B4) and (B5), Appendix B.] ( $a$, c) $\tau=43 \mathrm{~min}, h=5 \mathrm{~min} ;(b, d) \tau=45 \mathrm{~min}, h=10 \mathrm{~min}$. 
exponential culture (without a spread in generation times) is called the 'ideal age distribution', and is given by the following formula (Powell, 1956):

$$
\begin{array}{ll}
n(a)=(\ln 2) \times 2^{1-a} & \text { for } 0 \leqslant a \leqslant 1 \\
n(a)=0 & \text { for } a>1
\end{array}
$$

where $a$ is the cell age and $n(a)$ is the differential frequency $(\mathrm{d} N / \mathrm{d} a)$ of cells in the population having the age $a$; the sum of all frequencies, i.e. the area under the distribution given by the above formula, is set at 1.0 (corresponding to $100 \%$ of the cells in the population). The formula shows that zero-age cells are twice as frequent as cells of the age $1 \cdot 0$, which reflects the fact that two zero-age cells are formed every time a $1 \cdot 0$-age cell divides. Due to the spread of generation times, however, the ideal age distribution does not occur in reality. Rather, some cells divide long before reaching the age $1 \cdot 0$, and others divide much later at an age greater than 1.0. Consequently, the age distribution does not abruptly end at age $1 \cdot 0$, but gradually decreases to zero, and with increasing age increasingly deviates from the ideal age distribution (Bremer \& Churchward, 1978). It is now possible to derive the actual (as opposed to the ideal) age distribution from the distribution of generation times in Fig. $4(a, b)$.

The distribution of generation times is equivalent to the kinetic curve that describes the rate of cell division in a synchronous culture (started from a zero-page population) for one cycle of division. Therefore, the time integral over the distribution of generation times describes the kinetic changes in the cell number, i.e. the synchronous growth curve, for one cycle of divisions (Fig. $4 c, d$ ). 'One cycle' means that every cell divides only once, whereas in an actual synchronous culture, overlapping growth curves from consecutive cycles, one mass doubling time apart, are superimposed. [The construction of the actual synchronous growth curve from such overlapping curves, each calculated from the parameters $h$ and $\tau$ as in Fig. $4(c, d)$, agrees exactly with observed growth curves; Bremer \& Chuang, 1981 $a$.] Using a previously derived theory (Bremer \& Churchward, 1978; see Appendix C for details), the age distributions for the cultures in Fig. 4, i.e. for $h=5 \mathrm{~min}$ and for $h=10 \mathrm{~min}$, were calculated from the one-cycle growth curves of Fig. $4(c, d)$. The results of this calculation are shown in Fig. 5 . The curves show the wide spread in the division ages.

According to the idealized distribution of $D$-periods in Fig. 1, the spread in the division ages is infinity. This means that there could be a small probability that long (filamentous), i.e. 'overaged', cells are generated that greatly lag behind the average division time in the culture. Whether such physiological filaments occur in reality is not known. This would depend on the question of whether or not the distribution of generation times could go beyond $2 \tau$ (or whether the age distribution could go beyond age $2 \cdot 0$ ), i.e. lastly, whether the $D$-period could become

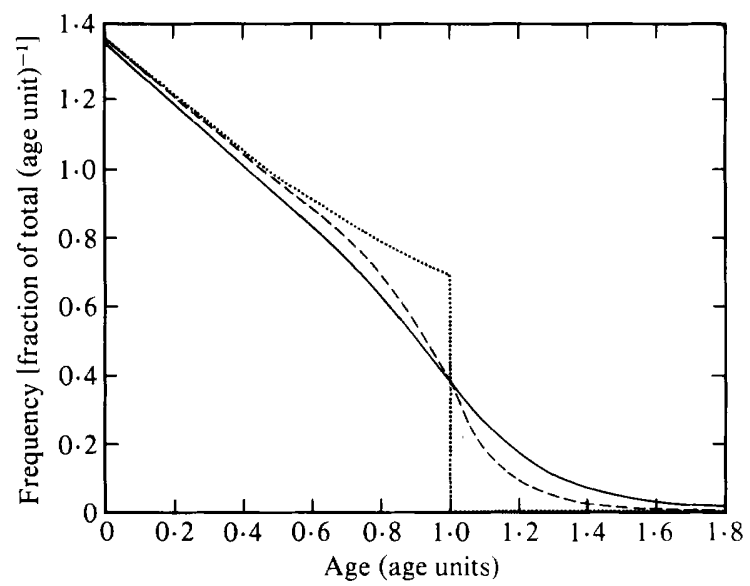

Fig. 5. Age distributions of exponential cultures for $\tau=45 \mathrm{~min}, h=10 \mathrm{~min}(-) ; \tau=43 \min , h=$ $5 \mathrm{~min}(---)$, and $h=0 \mathrm{~min}(\cdots \cdots)$. 
longer than $\tau$. It is conceivable that a delay of the signal for septum formation, caused by the random nature of signal generation, will always come to an end when the signal for the next division is generated; this would then lead to two or three simultaneous divisions of a cell around age 2 .

\section{Synchronous growth of cell populations with different replication phase relationships}

According to the preceding discussion, the phase relationships of a cell population can be completely described by two distributions, the distribution of division ages and the distribution of replication ages. In general, these distributions oscillate but, after long periods of growth, they tend to become stationary in a dynamic equilibrium. Figure 6 shows four examples of idealized age distributions: $(a)$ the distribution of a non-synchronous, exponential culture; $(b)$ the distribution at a given instant of a culture growing on the surface of an inverted membrane from which one of the two daughter cells in every division is always removed (age fractionation method used by Helmstetter \& Cooper, 1968); $(c)$ a distribution from a culture in which all ages are equally frequent - this distribution is obtained from $(b)$ after prolonged growth periods on the membrane when all cyclic fluctuations have disappeared; and $(d)$ the narrow age distribution of a synchronous culture. The distribution in $(c)$ may be regarded as the membrane equivalent of a steady-state exponential culture. Each of the four division age distributions in Fig. 6 may be associated with an infinite number of different replication age distributions. (Note that this is also true for the age distribution of an exponential culture, although in that case the population would have to be artificially mixed and could not be obtained simply by prolonged exponential growth; besides, growth would not be exponential in the case of an artificial mixture of a population which has the division age distribution, but not the replication age distribution, of an exponential culture.)

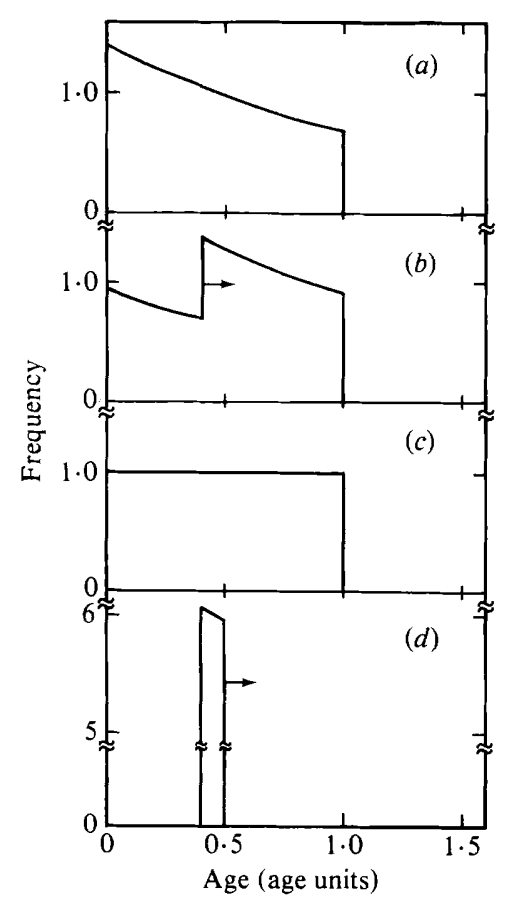

Fig. 6. Idealized age distributions of hypothetical cultures with invariable interdivision intervals $(h=$ $0)$. (a) Distribution of an exponential culture (Powell, 1956); (b) distribution of a culture growing on an inverted membrane (method of Helmstetter \& Cummings, 1964); $(c)$ same as $(b)$ after very long growth times when all age fluctuations have been blurred out; $(d)$ distribution of a synchronous culture. $\rightarrow$, Feature of distribution moves periodically from left to right with a cycle time of $\tau$. 


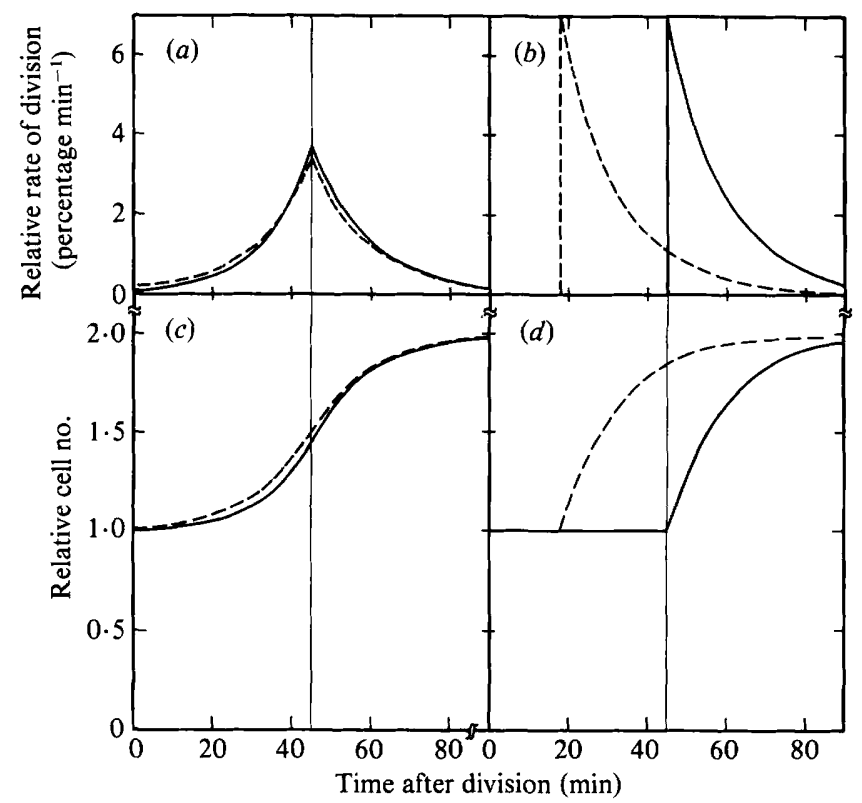

Fig. 7. Synchronous growth of zero-age populations of bacteria (doubling time $\tau=45 \mathrm{~min}$ and $h=$ $10 \mathrm{~min}$ ) with different phase distributions of replication cycles; $(a)$ and $(b)$ show rates of division and $(c)$ and $(d)$ (one-cycle) growth curves. $(a, c)$ Synchronous growth of a zero-age population, either from an exponential culture (-), or from an inverted membrane culture with an even age distribution [as in Fig. $6(c)](---)$. The first type of growth might be obtained by using the membrane elution technique of Helmstetter \& Cummings (1964) and eluting the bacteria from the membrane either immediately after the initial attachment (not practical due to presence of non-specifically bound cells in the eluate), or exactly $\tau$ min later. The second type of growth is obtained by using the membrane elution technique as before, but eluting the zero-age cells after very long growth on the membrane. $(b, d)$ Synchronous growth from a zero-age population with synchronized cycles of chromosome replication, such that termination has occurred either $18 \mathrm{~min}$ before zero time (-) or $45 \mathrm{~min}$ before zero time (----). Such populations could be obtained using size fractionation techniques (filtration or zonal sedimentation) and selecting and subculturing only the smallest cells present, which have divided $D_{0} \min$ after termination, or very large cells which have divided $45 \min (=\tau)$ after termination. In the latter case, termination of a new round of replication would coincide with the division at zero time.

To illustrate how different replication phase relationships affect the growth of synchronous cultures, Fig. 7 shows several examples of synchronous growth from cultures with the same division age distribution, but with different replication age distributions. In Fig. $7(a, c)$, the cell cycles are aligned at the zero-time division, but the replication cycles are not in phase; in this case the culture is thought to be obtained from a zero-age population either of an exponential culture [solid line, generation time distribution in Fig. 7(a) with skew] or from an inverted membrane culture as in Fig. 6(c) [dashed, symmetrical generation time distribution in Fig. 7(a)]. In Fig. $7(b, d)$, both replication and division ages are aligned at zero time, with either a phase difference of $D_{0}$ (solid line), i.e. all cells have terminated a round of replication at $D_{0} \min$ before zero time, or a phase difference greater than $D_{0}$ (dashed curves). This type of synchrony can be experimentally approximated by separating either the smallest cells (solid line) or larger cells of a given size (dashed curves) from a culture, using size fractionation methods, and using these size-fractionated cell populations to start a synchronous culture. Despite their differences, all four growth curves in Fig. $7(c, d)$ can be obtained from zero-age subpopulations of the same culture; thus, without taking into account the effects of replication phase relationships illustrated here, the curves might have been misinterpreted as showing effects of altered control of replication and cell division. 


\section{Table 1. Comparison of $\tau, B, C$ and $D$ with $\bar{\tau}, \bar{B}, \bar{C}$ and $\bar{D}$}

The parameters $\tau, B, C$ and $D$, defined for exponential cultures (Bremer \& Churchward, 1977), are compared with the average interdivision interval $(\bar{\tau})$, the average replication initiation time after division $(\bar{B})$, the average time to replicate the chromosome $(\bar{C})$, and the average time between termination of replication and the following division $(\bar{D})$. In this example, the parameter values used for the calculations were: $\tau=45 \mathrm{~min}, C=45 \mathrm{~min}, \Delta C= \pm 10 \mathrm{~min}$ (= variability in C-period), $D_{0}=18 \mathrm{~min}$, $h=10 \mathrm{~min}$.

\begin{tabular}{ccl} 
Parameter & $\begin{array}{c}\text { Duration } \\
\text { (min) }\end{array}$ & \multicolumn{1}{c}{ Equation used $^{*}$} \\
$\tau$ & $45 \cdot 0$ & $\bar{\tau}=\tau+h / \ln 2[(\tau / h)+1]^{\mathrm{a}}$ \\
$\bar{\tau}$ & $47 \cdot 6$ & $B=2 \tau-(C+D)^{\mathrm{b}}$ \\
$B$ & $14 \cdot 0$ & $\bar{B}=B_{0}-h / \ln 2[1+(h / \tau)] ; B_{0}=2 \tau-\left(C_{0}+D_{0}\right)^{\mathrm{c}}$ \\
$\bar{B}$ & $15 \cdot 2$ & \\
$C$ & $45 \cdot 0$ & $\bar{C}=C+[(\tau / \ln 2) \times(-\ln f)] ; f=2 /\left[2^{-\Delta C / \tau}+2^{+\Delta C / \tau}\right]^{\mathrm{d}}$ \\
$\bar{C}$ & $45 \cdot 8$ & $D=D_{0}+(\tau / \ln 2) \times \ln [1+(h / \tau)]^{\mathrm{e}}$ \\
$D$ & $31 \cdot 0$ & $\bar{D}=D_{0}+(h / \ln 2)^{\mathrm{f}}$
\end{tabular}

* Equations: (a) Equation (B3), this work; (b) Cooper \& Helmstetter (1968); (c) Equation (A3), this work; (d) Equation (A8) of Churchward et al. (1982); (e) Equation (B4) of Bremer \& Chuang (1981 b); (f) Equation (B2) of Bremer \& Chuang $(1981 b)$.

\section{Average values of cell cycle parameters}

Another implication of the variability of $D$-periods is a complication arising in the definition of certain cell cycle parameters : the $B$-period, $C$-period, $D$-period and $\tau$, describing chromosome replication initiation, termination, cell division and mass increase ('growth'). The value of any one of these four parameters is implied in the values of the other three (see below). For these parameters, two kinds of average values can be defined: (1) Averages denoted by $\bar{B}, \bar{C}, \bar{D}$ and $\bar{\tau}$ are the sums of individual values observed divided by the number of values summed (e.g. $\bar{B}=$ $\left.\sum B_{\mathrm{i}} / n\right) ;(2)$ Averages denoted by $B, C, D$ and $\tau$ (without the bar) are derived from exponential cultures (Bremer \& Churchward, 1977): if the exponential increase with time $(t)$ in the number of cells is given by the function $2^{t / \tau}$, then $\tau$ is defined by this function, and the number of chromosomal replication termini and origins in this culture can be described by the functions $2^{(t+D) / \tau}$ and $2^{(t+C+D) / \tau}$, respectively, i.e. by functions that are horizontally shifted against the cell number curves by $D$ or $(C+D)$ min, respectively, which thus define $C$ and $D$. $B$ is defined analogously as the horizontal (time) distance between the functions $2^{(t+n \tau) / \tau}$ and $2^{(t+C+D) / \tau}$, where $n$ has an integer value such that $\tau \leqslant(C+D) \leqslant n \tau$.

With either definition, the following analogous relationships exist between the four parameters : $\bar{B}+\bar{C}+\bar{D}=n \bar{\tau}$ or $B+C+D=n \tau$ (Cooper \& Helmstetter, 1968). $B$ and $\bar{B}, C$ and $\bar{C}$, etc., are compared in Table 1 . The equations in Table 1 define the relationships between $\tau$ and $\bar{\tau}$, $B$ and $\bar{B}$, etc. The equations show that the differences between the two kinds of averages disappear for zero variability $(h \rightarrow 0 ; \Delta C \rightarrow 0)$. The example in Table 1 also shows that the differences between $\tau$ and $\bar{\tau}, B$ and $\bar{B}$, etc., are only a few percent; i.e. in practice, these differences are negligible, but for any theoretical treatment of the cell cycle they must be taken into account.

This work was supported by the National Institutes of Health, grants GM15142 and GM25948. I thank Sally Rahn for her patience in typing this manuscript.

\section{A P PENDIX}

\section{(A) Distribution of replication initiation times}

We consider a hypothetical synchronous culture obtained as a zero-age population from an exponential culture. This synchronous culture contains a particular subpopulation in which a round of replication was terminated exactly $D_{0}$ min before zero time (Fig. 2); in these cells, a 
new round of replication will be initiated at the time $B_{0}$ after zero time (Fig. 3). The value of $B_{0}$ is found from the following relation (Cooper \& Helmstetter, 1968):

$$
B_{0}+C_{0}+D_{0}=n \tau
$$

where $n$ has an integer value, and $C_{0}$ is the $C$-period (chromosome replication time) at maximum replication velocity (i.e. for thymine-requiring bacteria the $C$-period observed at high concentrations of exogenous thymine). For example, for $E$. coli $\mathrm{B} / \mathrm{r}$ growing in glucose minimal medium at $37^{\circ} \mathrm{C}, C_{0}=45 \mathrm{~min}, D_{0}=18 \mathrm{~min}, \tau=43 \mathrm{~min}, n=2$, we find

$$
B_{0}=2 \times 43-45-18=23 \mathrm{~min}
$$

The rate of initiation $(\mathrm{d} I / \mathrm{d} t)$ in the synchronous culture increases exponentially until $t=B_{0}$, then it drops to zero (Fig. 3). The exponential increase occurs in proportion to the product of the exponential functions $2^{t / \tau}$ and $2^{t / h}$ (Fig. 2, and text to Fig. 2):

$$
(\mathrm{d} I / \mathrm{d} t) \approx 2^{t / t} \cdot 2^{t / h}=2^{t / \tau_{\mathrm{i}}} \text { for } t<B_{0}
$$

where

$$
1 / \tau_{\mathrm{i}}=(1 / \tau+1 / h), \quad \text { or } \quad \tau_{\mathrm{i}}=h /[1+(h / \tau)]
$$

Normalizing this function such that the total initiations (from $t=-\infty$ to $t=B_{0}$ ) of one round replication are set at $1 \cdot 0$, we find:

$$
\left.\begin{array}{cc}
(\mathrm{d} I / \mathrm{d} t)=\left(\ln 2 / \tau_{\mathrm{i}}\right) \cdot 2^{\left(t-B_{0}\right) / \tau_{\mathrm{i}}} & \text { for } t \leqslant B_{0} \\
(\mathrm{~d} I / \mathrm{d} t)=0 & \text { for } t>B_{0}
\end{array}\right\}
$$

These equations describe the distribution illustrated in Fig. 3.

On the average, initiation occurs earlier than $B_{0}$, at the time $\bar{B}$ :

$$
\bar{B}=B_{0}-\left(\tau_{\mathrm{i}} / \ln 2\right)=B_{0}-\frac{h}{\ln 2[1+(h / \tau)]}
$$

The average initiation age $\left(\bar{a}_{\mathrm{i}}\right)$ is found by dividing equation (A3) by $\tau$ :

$$
\bar{a}_{\mathrm{i}}=\frac{B_{0}}{\tau}-\frac{1}{\ln 2[(\tau / h)+1]}
$$

For $E$. coli $\mathrm{B} / \mathrm{r}$ in glucose minimal medium, using $B_{0}=23 \mathrm{~min}$ (see above), $\tau=43 \mathrm{~min}, h=$ 5 min (Bremer \& Chuang, 1981 a), we find

$$
\bar{a}_{\mathrm{i}}=\frac{23}{43}-\frac{1}{\ln 2[(43 / 5)+1]}=0 \cdot 38
$$

This value is somewhat smaller than the value of 0.55 estimated by Cooper \& Helmstetter (1968).

\section{(B) Distribution of interdivision intervals}

The distribution of interdivision intervals (generation times of individual cells) is identical to the kinetics of the rate of cell division, $\mathrm{d} C / \mathrm{d} t$, in a synchronous culture during one cycle period. This rate is found from the distribution of termination times (assumed to be equal to the distribution of initiation times but shifted $C$ min to the right) and from the distribution of $D$ periods, using equation (A1) of Bremer \& Chuang (1981a). According to this equation, the maximum rate occurs $\left(C_{0}+D_{0}\right)$ min after the peak of the initiation curve at $B_{0}$, which is $\tau$ min after zero time (Fig. $4 a, b$ ). Before the maximum, i.e. for $0 \leqslant t \leqslant \tau$, the curve reflects the exponentially increasing termination rate with the doubling time $\tau_{\mathrm{i}}$ such that the rate is given by

$$
\frac{\mathrm{d} C}{\mathrm{~d} t}=\frac{\ln 2}{\tau_{\mathrm{i}}[2+(h / \tau)]} \cdot 2^{(t-\tau) / \tau_{\mathrm{i}}} \quad \text { for } t \leqslant \tau
$$


After the maximum, the curve reflects the decreasing section in the distribution of $D$-periods with the half-life time $h$ :

$$
\frac{\mathrm{d} C}{\mathrm{~d} t}=\frac{\ln 2}{\tau_{\mathrm{i}}[2+(h / \tau)]} \cdot 2^{-(t-\tau) / h} \text { for } t \geqslant \tau
$$

The common factor $\ln 2 / \tau_{\mathrm{i}}[2+(h / \tau)]$ is chosen such that the total area under the rate curve is $1 \cdot 0$, and that at $t=\tau$ the rates calculated from equations (B1) and (B2) are the same. The rate curves given by equations (B1) and (B2) are illustrated in Fig. 4(a,b).

The average interdivision interval $(\bar{\tau})$ is equal to ' $\tau$ minus the average deviation to the left plus the average deviation to the right of the maximum in the division rate curve' (Fig. $4 a, b$ ):

$$
\bar{\tau}=\tau-\frac{\tau_{\mathrm{i}}}{\ln 2}+\frac{h}{\ln 2}=\tau+\frac{h}{\ln 2\{1+[1 /(h / \tau)]\}}
$$

This shows that $\bar{\tau}$ is always greater than $\tau$.

The one-cycle growth curve of a synchronous culture [as defined in Appendix (A)] is the integral over the rate curve from equations (B1) and (B2):

$$
\begin{gathered}
C_{t}=\frac{1}{2+(h / \tau)} \cdot 2^{(t-\tau) / \tau_{\mathrm{i}}} \quad \text { for } t \leqslant \tau \\
C_{t}=\frac{1}{2+(h / \tau)}\left\{1-\frac{h}{\tau_{\mathrm{i}}}\left[2^{-(t-\tau) / h}-1\right]\right\} \text { for } t \geqslant \tau
\end{gathered}
$$

These curves are illustrated in Fig. $4(c, d)$.

\section{(C) Age distribution}

The age distribution of bacteria $[n(a)]$ in an exponential culture is related to the one-cycle growth curve of equations (B4) and (B5) [equation (6) of Bremer \& Churchward, 1978]:

$$
n(a)=\ln 2 \cdot 2^{1-a}\left[1-N_{a}\right]
$$

Where $N_{a}$ is the one-cycle growth curve with the time measured relative to $\tau$, in age units [i.e., setting $t / \tau=a$ in equations (B4) and (B5)]:

$$
\begin{gathered}
N_{a}=\frac{1}{2+(h / \tau)} \cdot 2^{(a-1) /\left(\tau_{\mathrm{i} j} / \tau\right)} \quad \text { for } a \leqslant 1 \\
N_{a}=\frac{1}{2+(h / \tau)}\left\{1-\frac{h}{\tau_{\mathrm{i}}}\left[2^{-(a-1) /(h / \tau)}-1\right]\right\} \text { for } a \geqslant 1
\end{gathered}
$$

Two age distributions were calculated from observed values of $h$ and $\tau$, using equations (C1) to (C3) (Fig. 5).

\section{REFERENCES}

Bremer, H. \& ChUANG, L. (1981a). The cell cycle in Escherichia coli $\mathrm{B} / \mathrm{r}$. Journal of Theoretical Biology 88, 47-81.

Bremer, H. \& ChuANG, L. (1981 b). Cell division after inhibition of chromosome replication in Escherichia coli. Journal of Theoretical Biology 93, 909-926.

Bremer, H. \& ChurchWARD, G. (1977). An examination of the Cooper-Helmstetter theory of DNA replication and its underlying assumptions. Journal of Theoretical Biology 69, 645-654.

Bremer, H. \& Churchward, G. (1978). Age fractionation of bacteria by membrane elution: relation between age distribution and elution profile. Journal of Theoretical Biology 74, 69-81.
COOPer, S. \& Helmstetter, C. E. (1968). Chromosome replication and the division cycle of Escherichia coli B/r. Journal of Molecular Biology 31, 519-540.

Churchward, G., BREMer, H. \& Young, R. (1982). Macromolecular composition of bacteria. Journal of Theoretical Biology 94, 651-670.

GudAs, L. \& PARDEE, A. (1974). Deoxyribonucleic acid synthesis during the division cycle of Escherichia coli; a comparison of strains $\mathrm{B} / \mathrm{r}, \mathrm{K}-12,15$, and $15 \mathrm{~T}^{-}$ under conditions of slow growth. Journal of Bacteriology 117, 1216-1223.

HaR VeY, J. D. (1972). Parameters of the generation time distribution of Escherichia coli $\mathrm{B} / \mathbf{r}$. Journal of General Microbiology 70, 109-114. 
Helmstetter, C. E. \& CoOper, S. (1968). DNA synthesis during the division cycle of rapidly growing Escherichia coli B/r. Journal of Molecular Biology 31, 507-518.

Helmstetter, C. E. \& Cummings, D. J. (1964). An improved method for the selection of bacterial cells at division. Biochimica et biophysica acta 82, 608-61 0 .

KEPES, F. \& KEPES, A. (1980). Synchronization automatique de la croissance de Escherichia coli. Annales de microbiologie 13, 3-16.

KUBITSCHEK, H. E. (1961). Normal distribution of cell generation rate. Experimental Cell Research 26, 439 450.

Mitchison, J. M. \& Vincent, W. S. (1965). Preparation of synchronous cell cultures by sedimentation. Nature 205, 987-989.

POWELl, E. O. (1956). Growth rate and generation time of bacteria, with special reference to continuous culture. Journal of General Microbiology 15, 492--511.

Powell, E. O. (1958). An outline of the pattern of bacterial generation times. Journal of General Microbiology 18, 382-417.
Powell, E. O. \& Errington, F. P. (1963). Generation times of individual bacteria: some corroborative measurements. Journal of General Microbiology 31, 315-327.

Schaechter, M., Williamson, J. P., Hood, J. R. \& KoCH, A. L. (1962). Growth, cell and nuclear divisions in some bacteria. Journal of General Microbiology 29, 421-434.

Shehata, T. E. \& MARR, A. G. (1970). Synchronous growth of enteric bacteria. Journal of Bacteriology 103, 789-792.

Smith, J. A. \& Martin, L. (1973). Do cells cycle? Proceedings of the National Academy of Sciences of the United States of America 70, 1263-1267.

Woldringh, C. L., De Jong, M. A., VAN Den Berg, W. \& KOPPES, L. (1977). Morphological analysis of the division cycle of two Escherichia coli substrains during slow growth. Journal of Bacteriology 131, 270 279. 